\title{
Trajectories of Perceived Parental Psychological Control across Adolescence and Implications for the Development of Depressive and Anxiety Symptoms
}

\author{
Adam A. Rogers \\ Laura M. Padilla-Walker \\ Brigham Young University, laura_walker@byu.edu \\ Ryan D. McLean \\ Jeffrey L. Hurst
}

Follow this and additional works at: https://scholarsarchive.byu.edu/facpub

Part of the Social and Behavioral Sciences Commons

\section{Original Publication Citation}

Rogers, A. A., Padilla-Walker, L. M., *McLean, R., \& *Hurst, J. (2020). Trajectories of perceived parental psychological control across adolescence and implications for the development of depressive and anxiety symptoms. Journal of Youth and Adolescence, 49, 136-149.

\section{BYU ScholarsArchive Citation}

Rogers, Adam A.; Padilla-Walker, Laura M.; McLean, Ryan D.; and Hurst, Jeffrey L., "Trajectories of Perceived Parental Psychological Control across Adolescence and Implications for the Development of Depressive and Anxiety Symptoms" (2019). Faculty Publications. 4972.

https://scholarsarchive.byu.edu/facpub/4972

This Peer-Reviewed Article is brought to you for free and open access by BYU ScholarsArchive. It has been accepted for inclusion in Faculty Publications by an authorized administrator of BYU ScholarsArchive. For more information, please contact ellen_amatangelo@byu.edu. 


\title{
Trajectories of Perceived Parental Psychological Control across Adolescence and Implications for the Development of Depressive and Anxiety Symptoms
}

\author{
Adam A. Rogers ${ }^{1} \cdot$ Laura M. Padilla-Walker $^{1} \cdot$ Ryan D. McLean $^{1} \cdot$ Jeffrey L. Hurst ${ }^{1}$ \\ Received: 18 June 2019 / Accepted: 24 June 2019 / Published online: 4 July 2019 \\ (c) Springer Science+Business Media, LLC, part of Springer Nature 2019
}

\begin{abstract}
Theory and research indicate considerable changes in parental control across adolescence (e.g., declining behavioral control), but the developmental course and significance of psychological control remains largely unknown. This study examined trajectories of adolescents' reports of mothers' and fathers' psychological control from ages 12 to 19, predictors of occupying distinct trajectories, and the developmental significance of these trajectories for adolescents' development of depressive and anxiety symptoms. It used eight waves of survey data on 500 adolescents $\left(M_{\text {age }}=11.83, S D=1.03 ; 52 \%\right.$ female; $67 \%$ White, $12 \%$ African American) and their parents from the Pacific Northwest United States. Most adolescents (about 90\%) reported low but increasing levels of parental psychological control over time, with a small but significant subset (about 10\%) perceiving perpetually elevated levels. Mothers' (but not fathers') depressive symptoms, reported at the age 12 assessment, predicted adolescents' membership in the elevated psychological control trajectory. Adolescents occupying these elevated trajectories showed more problematic growth in depressive and anxiety symptoms across adolescence. Taken together, the findings suggest that many adolescents experience increased parental psychological control as they age, and that variability in these trends indicates individual differences in their development of depressive and anxiety symptoms over time.
\end{abstract}

Keywords Psychological control $\cdot$ Parents $\cdot$ Adolescents $\cdot$ Trajectories

\section{Introduction}

The landscape of parental control changes considerably across adolescence as children grow in maturity and independence (Keijsers and Poulin 2013). However, little remains known about how adolescents' experiences of psychological control change across the adolescent years. Parental psychological control refers to a set of subtle and manipulative parenting strategies aimed at coercing adolescents into ways of thinking and feeling that effectively mirror their parents (Barber 1996). Psychological control is

Supplementary information The online version of this article (https:// doi.org/10.1007/s10964-019-01070-7) contains supplementary material, which is available to authorized users.

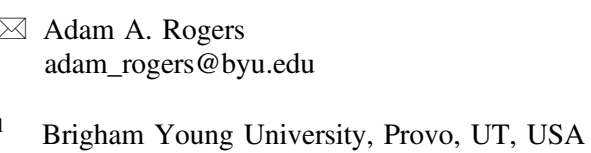

distinct from behavioral control, which typically involves overt attempts to regulate adolescent behavior, for example by establishing rules and boundaries, enacting consistent discipline when rules are violated, and monitoring adolescent activities (e.g., Dishion and McMahon 1998). In contrast, psychological control uses covert strategies such as guilt induction, conditional regard, love withdrawal, and dismissiveness, in an attempt to leverage adolescents' internal (psychological and emotional) states to get them to "coerce, manipulate, or control themselves" (Soenens and Vansteenkiste 2010, p. 80). Although harmful in general, psychological control is especially problematic during adolescence because it interferes with autonomy development and promotes continued dependence on parents (Soenens and Vansteenkiste 2010). Indeed, adolescents' experiences of psychological control predict numerous challenges, and particularly internalizing problems such as depression and anxiety (e.g., Werner et al. 2016). Therefore, unpacking the developmental etiology of parental psychological control is needful for informing family-based 
prevention and intervention efforts that promote adolescent well-being. This study examined the developmental course of adolescents' perceived psychologically controlling parenting from early to late adolescence, and its associations with adolescents' development of depressive and anxiety symptoms.

\section{The changing landscape of parental control}

As adolescents increase in autonomy and independence, they spend more time outside the home and communicate less frequently with parents (Keijsers and Poulin 2013). Disagreements also arise regarding a parent's right to know about adolescents' personal lives (Smetana et al. 2006) and adolescents increasingly assert boundaries by actively managing information that is and is not made available to parents (e.g., Dietvorst et al. 2018). As a result, parental knowledge diminishes steadily over the adolescent years, and behavioral control strategies (e.g., monitoring) that once provided high levels of routine and structure become less feasible for regulating adolescents' decisions and behaviors, and therefore less frequent (Keijsers and Poulin 2013). Although reasonable levels of parental knowledge and behavioral control remain protective throughout adolescence (Dishion and McMahon 1998), these trends suggest a normative pattern of individuation that is appropriate to the degree that they are paced with the developmental competencies of the adolescent.

Considerably less is known about how parental psychological control may change across these same years. From the extant literature, it seems that adolescents are increasingly likely to perceive or experience psychological control as they become more independent. With the greater salience of an identity and sense of self, adolescents may become more sensitive to parents' infringements upon their independence. Parents may also legitimately employ psychologically controlling strategies more frequently in the home when they perceive that they are gradually losing their ability to directly control and regulate adolescent behavior through rules and disciplinary means. In the context of these changes, parents may begin to prefer the more covert strategies of psychological control as a more feasible way of exerting influence. Furthermore, because psychological control may be a response to parenting stress, and particularly aversive parent-child dynamics (Steeger and Gondoli 2013), the increasing intensity of parent-child conflict during the adolescent years could elicit more psychological control.

Unfortunately, these suppositions have yet to be empirically examined, as very little data is available to inform conclusions about the developmental course of perceived psychological control across the second decade of life. To date, Meter et al. (2019) have provided perhaps the only such investigation of its kind, showing low and stable levels of parent-reported psychological control across the high school years (grades 9-12). Although informative, parentreported psychological control may have relatively little overlap with adolescents' own experiences of such (e.g., De Los Reyes et al. 2009). Furthermore, adolescents' own perceptions of parenting are arguably more representative of their lived experiences, and likely are more predictive of their adjustment outcomes (e.g., Van Lissa et al. 2019). Therefore, examinations of how adolescents' perceive parental psychological control to change over time is needed.

Some studies have examined development in adolescent reported parental psychological control, but these have focused on the late-adolescent or young adult period. As such, they speak primarily to periods when many adolescents have already left the home, autonomy shifts have nearly completed, and family roles re-stabilized. These studies have shown perceived psychological control to be relatively stable from ages 18-20 (Luyckx et al. 2007) or even decline between ages 19-25 (Desjardins and Leadbeater 2017). Ultimately, there is little research to inform these processes from early- to lateadolescence, despite this being a sensitive period for the emergence of family conflict and adjustment challenges. Therefore, this study sought to examine the developmental course of adolescents' perceptions of mothers' and fathers' psychological control across an 8-year period spanning early to late adolescence (ages 12-19).

Recent studies have indicated striking heterogeneity in individuation-related family processes throughout adolescence, signaling that the ways in which families navigate autonomy transitions is quite individualized (e.g., Keijsers et al. 2016). It is reasonable, then, to anticipate meaningful individual differences in psychological control trajectories across adolescence. Person-centered analytics adapt elegantly to longitudinal data, capturing between-family differences in within-family patterns of growth and change. The application of such an approach to the development of psychological control can be particularly valuable in helping identify those families who might be at particular risk. Indeed, it is probable that not all families start at the same place or change similarly over time in regards to their psychologically controlling dynamics, and these differences can be modeled and tested empirically. Therefore, the current study took an additional person-centered approach using longitudinal mixture modeling to identify distinct trajectories of mothers' and fathers' psychological control across adolescence (ages 12-19).

\section{Parent and child predictors of psychological control trajectories}

The second aim of this study was to explore antecedents of psychological control trajectories that could inform understanding of risk factors for elevated psychological control 
across the second decade. Both parent- and child- characteristics have been shown to operate on psychological control, but infrequently have these been examined in tandem or in relation to long-term psychological control trajectories. Studies examining parent predictors of psychological control point to the effects of parents' own internalizing challenges, particularly depressive symptoms. It is well established that parents (especially mothers) who are depressed tend to provide lower quality parenting environments characterized by less sensitivity and responsiveness, and elevated levels of harshness (e.g., Taraban et al. 2017). Unsurprisingly, then, studies have linked mothers' depressive symptoms with their own psychologically controlling behavior (Cummings et al. 2005), a link that is understood to reflect a parents' felt need to maintain some degree of control in family life (Pettit et al. 2001). Therefore, this study examined whether parents' depressive symptoms might predict adolescents' membership in different psychological control trajectories spanning early to late adolescence.

Child factors may also predict more psychologically controlling parenting and/or the adolescent's perception of such. Although these factors are not studied extensively, the available research points particularly to adolescents' externalizing behaviors. This is because externalizing behaviors, such as aggression and delinquency, can provoke more conflictual family dynamics that then elicit heightened control attempts by parents, including psychological control. This process is documented in recent longitudinal work. For example, one study found that adolescent aggression in 6th grade was associated with motheradolescent conflict in 7th grade, which was in turn associated with higher levels of mother- and adolescent-reported psychological control in 8th grade (Steeger and Gondoli 2013). Another longitudinal, cross-lagged study found that there were bidirectional links between adolescent reports of psychological control and parent-reported adolescent aggression from age 13-16, suggesting that children's externalizing behavior and psychological control may exacerbate one another over time (Janssens et al. 2017). Therefore, this study also examined whether adolescents' externalizing behaviors could indicate membership in psychological control trajectories across adolescence.

\section{The developmental significance of parental psychological control for depressive and anxiety symptoms}

Finally, the third aim of this study was to explore the developmental significance of following distinct trajectories of psychological control for adolescent internalizing problems. The second decade of life ushers in a heightened vulnerability to such challenges, and a robust literature implicates parental psychological control as one risk factor for these problems. Adolescents' experiences of a psychologically controlling home environment are more than just dysregulating (e.g., Yap et al. 2008) but actually undermine autonomous functioning by inducing coerced states of functioning (Soenens and VanSteenkiste 2010). These coerced states can deplete psychological resources, such as active self-control (Rogers et al., under review), and as a result, adolescent reports of their parents' psychological control robustly predict their higher internalizing problems, such as depressive symptoms (Soenens et al. 2008) and lower self-esteem (Wang et al. 2007). Currently, however, most studies linking psychological control to indices of mental health are cross sectional or short-term longitudinal, and as such, they tend to focus on relatively narrow developmental periods, rather than examining psychological control and mental health across development. Thus, the current study explored how differential trajectories of psychological control relate to adolescents' developmental trends (within-person change) of depressive and anxiety symptoms across adolescence and into the transition to adulthood, a span in which autonomy needs are especially salient and vulnerabilities to internalizing distress are heightened.

\section{Current study}

The goals of the present study were three-fold. First, it examined overall developmental trends in adolescents' perceptions of maternal and paternal psychological control from ages 12-19, which were predicted to increase across adolescence. It then investigated between-family heterogeneity in these developmental trends, specifically seeking distinct classes or profiles of within-person change in perceived psychological control. Given the exploratory nature of these modelling techniques and the paucity of extant literature, hypotheses here were also exploratory. Second, this study examined evidence for both parent- and childdriven effects on these trajectories, specifically investigating whether parent depressive symptoms and children's externalizing behaviors at age 12 might predict membership in these distinct trajectories. Finally, this study explored how following these distinct trajectories of perceived psychological control differentially predicted the development (within-person change over time) of depressive and anxiety symptoms across adolescence (ages 14-19). It was hypothesized that adolescents who followed more elevated trajectories of perceived psychological control would display more elevated levels of depressive and anxiety symptoms across development. 


\section{Method}

\section{Participants and procedures}

Data for this study came from the Flourishing Families Project, a 10-year longitudinal study of family life and adolescent development. In 2007, after receiving ethics approval from the Brigham Young University Institutional Review Board, families were recruited using a purchased national telephone survey database (Polk Directories/ InfoUSA) containing 82 million households across the United States. Families were eligible to participate if they had a child between the ages of 10 and 14 and lived in targeted census tracts in the Pacific Northwest, USA. Using the Polk Directory, families were randomly selected from census tracts that paralleled the socio-economic and racial/ ethnic makeup of local school districts. A total of 692 eligible families were contacted, 423 of which agreed to participate. An additional subset of families $(n=77)$ were recruited via referrals. Then, parental consent and adolescent assent were obtained, after which the 500 participating families (i.e., each parent and the focal child) were interviewed in their homes once per year for the first five years (waves 1-5). These interviews included video-taped interaction tasks and questionnaires about family life and adjustment indices. Over the next five years (Waves 6-10), only questionnaire data were collected, and were done so via online surveys. The final wave of data was collected in 2016.

Participants for the present study included all 500 participating families, including target adolescents and their parents. In total $90 \%$ of the families were retained for all ten waves of the study. At the first assessment, adolescents were an average age of $11.83(S D=1.03)$, were roughly equal on sex (52\% Female), and represented non-Hispanic Caucasian $(n=67 \%)$, African American $(n=12 \%)$, Hispanic/Latinx $(n=2 \%)$, Asian American $(n=4 \%)$, and other $(n=15 \%)$ backgrounds. Parents reported an average income level of $\$ 68,735.65$; mothers were an average age of 43.18 years and fathers an average of age 45.32 years. For the present study, these data were re-structured by adolescent age so that the estimation of growth curves would corresponded to age (instead of wave), and therefore more accurately track a developmental process. This created some missing data at the extreme ends of the age range in the sample (age 11; age 20). Therefore, this study used the eight assessments for each family during which their adolescent was between 12 and 19 years, representing the most robust data coverage across families while still capturing the processes of interest from early adolescence to late adolescence.

\section{Measures}

\section{Parental psychological control}

At each assessment, adolescents reported on their mothers' and fathers' psychological control using the Psychological Control-Youth Self Report scale (PC-YSR; Barber 1996), which is a widely used measure of perceived psychologically controlling parenting. For each parent, adolescents were presented with 8 items, such as "my parent is less friendly with me if I do not see things her/his way" and "my parent brings up past mistakes when s/he criticizes me". Adolescents responded on a 5-point Likert-type scale $(1=$ Never, $5=$ Always). Across the eight assessments, Cronbach's Alphas ranged from $0.85-0.91$ for perceived mothers' psychological control and $0.82-0.91$ for perceived fathers' psychological control.

\section{Adolescent externalizing behaviors}

At the age 12 assessment, parents reported on their children's externalizing behaviors. Externalizing behaviors were measured using problem-behavior and delinquencyrelated items (Barber et al. 2005a, 2005b), albeit with phrasing adaptations to allow for parental responses about their children. Sample items include "My child lies or cheats" and "My child steals things from places other than home." Internal consistency was $\alpha=0.70$ for mothers and $\alpha=0.71$ for fathers.

\section{Parent depressive symptoms}

When their adolescents were 12 years old, parents also completed a shortened version of the Center for Epidemiologic Studies-Depression scale (CESD; Radloff 1977). The stem "How often did this happen in the past week" was followed by 11 items, such as "I felt everything I did was an effort" and "I felt lonely". Items were rated on a 3-point likert scale $(1=$ never, $2=$ some of the time, $3=$ most of the time). Internal consistency was $\alpha=0.79$ for mothers and $\alpha$ $=0.77$ for fathers.

\section{Adolescent depressive symptoms}

From ages 14 to 19 , adolescents completed annual assessments of the Center for Epidemiological Studies-Depression for Children scale (CES-DC; Weissman et al. 1980). This widely used scale uses 20 items mirroring the original CES-D but with wording adaptations suited for children and adolescents. Numerous studies have indicated its internal reliability, construct validity, and positive and negative 
predictive value (see Stockings et al. 2015). The stem "during the past week" was followed by 20 items, such as "I felt everything I did was an effort," "I felt down and unhappy", and "I felt like something bad was going to happen." Items were rated on a 4-point Likert scale $(1=$ not at all, $2=$ a little, $3=$ some, $4=a$ lot $)$. Internal consistency ranged from $\alpha=0.89$ to 0.94 .

\section{Adolescent anxiety symptoms}

From ages 14 to 19 , adolescents completed the six item generalized anxiety disorder subscale from the Spence Child Anxiety Inventory (Spence 1998), for which construct validity has been established among adolescent populations in prior studies (e.g., Essau et al. 2002). Sample items included "I worry a lot about things," and "When I have a problem, my heart beats really fast." Items were scored on a 4-point Likert-type scale $(0=$ never, $3=$ always $)$. Alphas ranged from $\alpha=0.83$ to 0.88 from ages $14-19$.

\section{Demographic controls}

Information was collected on family structure $(1=2$ Parent married family, $2=$ Single parent family) parents' education level $(1=$ Less than High School, $2=$ High School, 3 = Some college, $4=$ Associates, $5=$ Bachelors, $6=$ Mas ters, $7=$ Advanced Degree $)$, adolescents' sex $(0=$ female, $1=$ male $),$ and adolescents' ethnicity $(1=$ European American, $2=$ African American, $3=$ Hispanic, $4=$ Asian American, $5=$ Other, $6=$ Multi-Ethnic). For purposes of analysis, ethnicity was dummy coded to represent ethnic minority status $(0=$ non-White Minority, $1=$ Non-Hispanic Caucasian).

\section{Analytic strategy}

First, study variables were screened for univariate outliers (defined as \pm 3.29 standard deviations from the mean) and missing data patterns. Then, descriptive statistics and correlations were calculated to examine preliminary data patterns.

\section{Trajectories of perceived psychological control across adolescence}

To test the first prediction regarding developmental change in parental psychological control from ages 12-19, a latent growth curve was estimated in a structural equation modelling (SEM) framework, centering the model at age 12 . Model building procedures were used to determine the best fitting trend, starting with a no-growth model (intercept only), followed by the addition of a linear slope, and then the addition of a quadratic term. The best-fitting model among these was retained, using the Akaike Information Criterion (AIC; Akaike 1973) and Bayesian Information Criterion (BIC; Akaike 1981) as indicators of model fit. These fit indices are comparative fit indices, having no inherent scaling and are used primarily for comparing nested models, lower values indicating better fit. All models controlled for adolescents' sex, ethnic minority status, parents' education, and family structure, trimming those that were not significant at $p<0.10$.

Once a normative trend was determined, identifiable subgroups were explored for trajectories of psychological control from ages $12-19$ by estimating growth mixture models (GMM). Because of the exploratory nature of GMM, several solutions were tested with different numbers of classes, and considered a number of fit and convergence indices together to arrive at the most appropriate solution. The best fitting solution would need to have a comparatively low Bayesian Information Criterion. It would also need to produce a statistically significant Lo-Mendell-Rubin likelihood ratio test (LMR-LRT), which compares a solution with $k$ classes to the prior solution with $k-1$ classes, testing the null hypotheses that $k$ does not fit the data better than $k-1$. In addition, this solution would need to display confident case classification, indicated by entropy values $>$ 0.80 (Grimm et al., 2017). The estimation of the solutions would need to be stable, ensured by first running models with 60 random starts and 10 final stage optimizations, and then 600 random starts and 100 final stage optimizations (Asparouhov and Muthén 2012). Finally, class sizes had to be large enough to be statistically robust and had to be theoretically meaningful.

\section{Parent and child antecedents of class membership}

Once appropriate classes were determined, antecedents of these classes were examined using the "3-step approach" in Mplus (Muthén and Muthén 1998-2018). This approach regressed class membership onto parents' depressive symptoms and adolescents' externalizing behaviors at age 12 , as well as all demographic controls (child sex, minority status, parents' education level, and family structure). A partialled odds ratio was produced for each predictor, representing the respective change in the likelihood of occupying each class.

\section{Class differences in development of depressive and anxiety symptoms}

The final phase of analysis examined whether class membership would differentiate adolescents in regards to their development of depressive and anxiety symptoms across adolescence. Because these measures were included at the third wave of the study, data coverage at years 12 and 13 
was particularly sparse. Therefore, growth curves were estimated for the six years spanning ages 14-19. Model building procedures were conducted to identify the best fitting growth solution for depressive and anxiety symptoms across ages 14-19 (e.g., no growth, linear, quadratic, etc). Then, the posterior probabilities used to determine class membership in the GMMs were entered as predictors of the growth parameters (e.g., intercepts, slopes, and quadratic terms). Posterior probabilities were used instead of distinct class membership because they account for the uncertainty in the classification process, assigning adolescents a probabilistic class membership. These models additionally controlled for adolescents' sex, minority status, family structure, and parent education level. Separate prediction models were estimated for depressive symptoms and anxiety symptoms. However, to more thoroughly understand the developmental processes of depressive and anxiety symptoms among the classes, these prediction models were estimated twice: once with the intercept centered at early adolescence (age 14) and another in which the intercept was centered at late adolescence (age 19). Re-centering the growth trends enables precise estimates of individual differences in development of depressive or anxiety symptoms at specific points of theoretical interest, and to examine whether such individual differences are sustained across development (King et al. 2017). Analyses were conducted in Mplus version 8.0 (Muthén and Muthén 1998-2018).

\section{Results}

\section{Data screening}

There were occasional univariate outliers beyond \pm 3.29 standard deviations, but as there were never outliers on more than two measurement occasions, all cases were retained for analyses. Missing data patterns were analyzed on the major variables of the study. Little's MCAR test (Little 1988) was, $X^{2}(10,308)=11,628.5, p<0.001$, leading to the rejection of the null hypothesis that the missing values were missing completely at random. Therefore, codes for missingness were created for each variable $(0=$ non-missing, $1=$ missing) and logistic regressions were used to predict missingness based off of parent education, ethnicity, and adolescents' sex. Parent education as well as ethnicity predicted missing data on fathers' depression and anxiety at age 12 . For this reason, parent education, ethnicity, and adolescent sex were included as covariates in the final models, and Full Information Maximum Likelihood was used to handle cases with missing data (FIML; Enders 2010).
Table 1 Means and standard deviations for mothers' and fathers' psychological control (ages 12-19) and adolescents' depressive and anxiety symptoms (ages 14-19)

\begin{tabular}{|c|c|c|c|c|c|c|c|c|}
\hline \multirow[t]{2}{*}{ Age } & \multicolumn{2}{|c|}{$\begin{array}{l}\text { Mothers' } \\
\text { psych } \\
\text { control }\end{array}$} & \multicolumn{2}{|c|}{$\begin{array}{l}\text { Fathers' } \\
\text { psych } \\
\text { control }\end{array}$} & \multicolumn{2}{|c|}{$\begin{array}{l}\text { Depressive } \\
\text { symptoms }\end{array}$} & \multicolumn{2}{|c|}{$\begin{array}{l}\text { Anxiety } \\
\text { symptoms }\end{array}$} \\
\hline & $M$ & $S D$ & $M$ & $S D$ & $M$ & $S D$ & $M$ & $S D$ \\
\hline 12 & 1.75 & 0.69 & 1.69 & 0.62 & & & & \\
\hline 13 & 1.82 & 0.68 & 1.75 & 0.69 & & & & \\
\hline 14 & 1.91 & 0.71 & 1.81 & 0.69 & 1.67 & 0.53 & 0.92 & 0.57 \\
\hline 15 & 2.02 & 0.78 & 1.87 & 0.75 & 1.73 & 0.64 & 0.98 & 0.61 \\
\hline 16 & 2.05 & 0.82 & 1.85 & 0.72 & 1.81 & 0.59 & 1.10 & 0.63 \\
\hline 17 & 2.08 & 0.83 & 1.85 & 0.74 & 1.81 & 0.60 & 1.17 & 0.66 \\
\hline 18 & 2.11 & 0.81 & 1.89 & 0.77 & 1.78 & 0.60 & 1.19 & 0.69 \\
\hline 19 & 2.00 & 0.78 & 1.81 & 0.74 & 1.77 & 0.56 & 1.22 & 0.71 \\
\hline
\end{tabular}

Psychological Control scale range is 1-5. Depressive symptoms scale range is $1-4$. Anxiety symptoms scale range is $0-3$

\section{Descriptive statistics}

Means and standard deviations for key study variables are presented in Table 1. Correlations are presented in Appendix A. Adolescent reports of mothers' and fathers' psychological control were significantly correlated at each age ( $r$ ranged from 0.51 to 0.63 ). Mothers' and fathers' psychological control were significantly related to age 12 adolescent externalizing behaviors (mothers' $r=0.18$ fathers' $r=0.10)$, and adolescent depressive symptoms $(r$ ranged from 0.16 to 0.49$)$ and anxiety symptoms ( $r$ ranged from 0.13 to 0.48 ) from age 14-19. Parents' depressive symptoms were related to their psychological control at age 12 (mothers' $r=0.12$, fathers' $r=0.15$ ), all $p$ 's $<0.05$.

\section{Trajectories of perceived psychological control across adolescence}

For both mothers and fathers, a quadratic model was the best-fitting model to describe overall change in psychological control from ages 12-19 (see Table S1 in supplemental material for fit indices). Table 3 presents parameter estimates of mother and father models, and Fig. 1a, b display overall growth trends for mothers and fathers, respectively. Both mother and father models showed low initial levels of psychological control at age 12 (intercept), with a positive slope indicating a steady rise in psychological control across adolescence. A significant, negative quadratic term indicated that these levels peaked around late adolescence and then tapered. In the mother model, controls for mothers' formal education significantly predicted the mothers' intercept. Mothers with less formal education were 
reported by their adolescent children to have higher initial levels of psychological control at age $12(b=-0.06, p=$ 0.01 ) although these mothers did not change faster or slower across adolescence, as indicated by non-significant associations with the slope and quadratic term. In the father model, controls indicated that non-white fathers $(b=$ $-0.18, p<0.001)$, fathers with less formal education $(b=$ $-0.05, p=0.007)$, and fathers of boys $(b=0.13, p=0.02)$
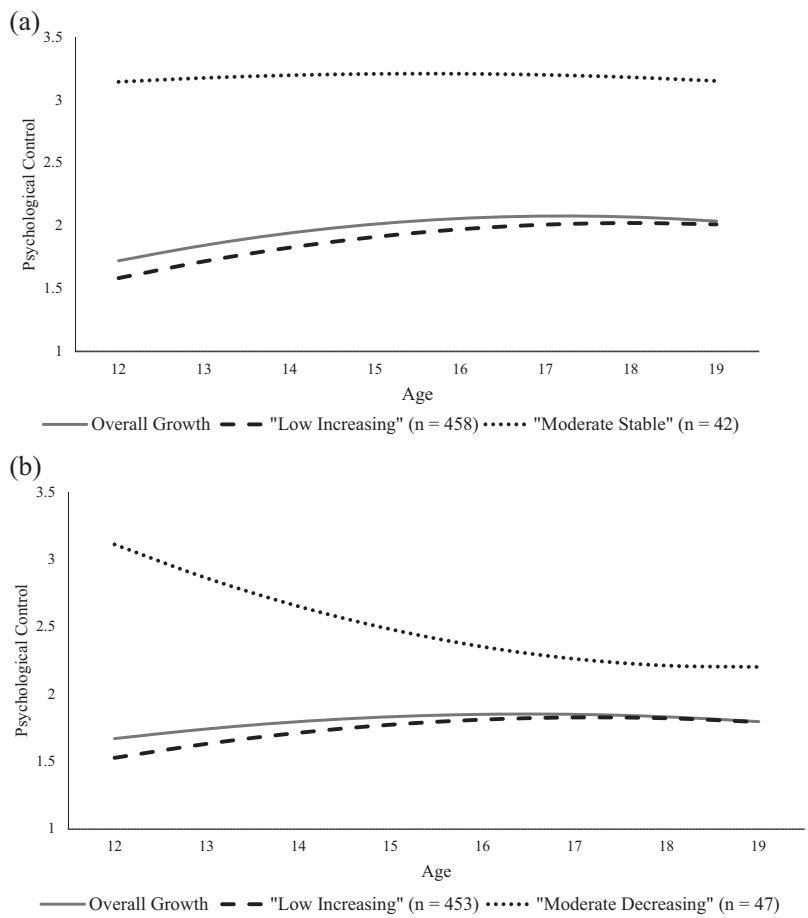

Fig. 1 Overall growth and distinct profiles of growth in adolescents' perceived (a) maternal and (b) paternal psychological control from ages 12-19 showed higher initial levels of psychological control at age 12 (although they did not change faster or slower across adolescence). Each of the growth parameters displayed significant variability, signaling the presence of potentially meaningful individual differences among trajectories of mothers' and fathers' psychological control.

Growth mixture modeling was used to explore if there were distinct subgroups of psychological control trajectories across ages 12-19. Several solutions were tested and compared across a variety of fit indices (see Table 2). For mothers, the 2- and 3-class solutions emerged as the most viable according to the indicators, showing the most acceptable patterns of model fit, classification, and meaningfulness. Of these, the 2-class solution was favored. Although the 3-class solution had slightly lower comparative fit (e.g., BIC values), its entropy was markedly lower and the LMR test failed to reject the null hypothesis that the 3 -class was identical in fit to the 2-class solution. Within this 2-class solution, the Class 1 mothers $(n=458,91 \%)$ showed low initial levels of psychological control, with a positive slope indicating a gradual increase across adolescence before peaking at age 17 and then tapering slightly, as indicated by a negative quadratic term (see Fig. 1a for plots; see Table 3 for parameter estimates). These mothers were called the "low increasing/normative" group, given that their trajectory largely mirrored the overall, normative trend from the prior analyses. Class 2 mothers $(n=48,9 \%)$ showed markedly higher initial levels of psychological control (intercept was moderate to high). The slope and quadratic terms were not significant, indicating that this group of mothers remained relatively stable in these elevated levels of psychological control across adolescence. These were called the "moderate stable" group. To gain further insight into the differences among these trajectories,
Table 2 Fit indices of GMM for mothers' and fathers' psychological control

\begin{tabular}{|c|c|c|c|c|c|c|c|c|}
\hline \multirow[t]{2}{*}{ Classes } & \multirow[t]{2}{*}{ BIC } & \multirow[t]{2}{*}{$\operatorname{LMR}(p)$} & \multirow[t]{2}{*}{ Entropy } & \multicolumn{5}{|c|}{ Class counts $(\%)$} \\
\hline & & & & Class 1 & Class 2 & Class 3 & Class 4 & Class 5 \\
\hline \multicolumn{9}{|l|}{ Mothers } \\
\hline 1 & 6954.245 & - & - & 100 & & & & \\
\hline 2 & 6869.997 & $104.89(0.01)$ & 0.92 & 90.99 & 9.01 & & & \\
\hline 3 & 6842.557 & $50.276(0.12)$ & 0.82 & 74.50 & 17.48 & 8.02 & & \\
\hline 4 & 6686.286 & $57.226(0.05)$ & 0.85 & 70.68 & 21.71 & 6.49 & 1.12 & \\
\hline 5 & 6816.818 & $27.849(0.23)$ & 0.86 & 72.08 & 14.93 & 9.55 & 2.32 & 1.12 \\
\hline \multicolumn{9}{|l|}{ Fathers } \\
\hline 1 & 6229.166 & - & - & 100 & & & & \\
\hline 2 & 6135.799 & $113.65(0.005)$ & 0.89 & 90.74 & 9.26 & & & \\
\hline 3 & 6072.022 & $85.21(0.12)$ & 0.89 & 82.16 & 14.29 & 3.55 & & \\
\hline 4 & 6043.131 & $54.034(0.14)$ & 0.88 & 78.35 & 11.34 & 7.20 & 3.10 & \\
\hline 5 & & & & Did not & & & & \\
\hline
\end{tabular}

$B I C=$ Bayesian Information Criterion; $L M R=$ Lo-Mendell-Rubin Adjusted Likelihood Ratio Test. Bolded rows represent those chosen as final solutions 
Table 3 Parameter estimates for each sub-trajectory of mothers' and fathers' 2-class solutions

\begin{tabular}{|c|c|c|c|}
\hline & Intercept & Slope & Quadratic \\
\hline \multicolumn{4}{|l|}{ Mothers } \\
\hline \multicolumn{4}{|c|}{ Overall Growth } \\
\hline Mean & $1.72 * * *$ & $0.14 * * *$ & $-0.01 * * *$ \\
\hline Variance & $0.29 * * *$ & $0.06 * * *$ & $0.001 * * *$ \\
\hline \multicolumn{4}{|c|}{ 2-Class Solution } \\
\hline \multicolumn{4}{|c|}{ Class $1(n=458)$} \\
\hline Mean & $1.58 * * *$ & $0.15 * * *$ & $-0.01 * * *$ \\
\hline Variance & $0.09 * *$ & $0.07 * * *$ & $0.001 * * *$ \\
\hline \multicolumn{4}{|c|}{ Class $2(n=42)$} \\
\hline Mean & $3.14 * * *$ & 0.04 & -0.02 \\
\hline Variance & $0.09 * *$ & $0.07 * * *$ & $0.001 * * *$ \\
\hline \multicolumn{4}{|l|}{ Fathers } \\
\hline \multicolumn{4}{|c|}{ Overall Growth } \\
\hline Mean & $1.66^{* * *}$ & $0.08 * * *$ & $-0.01 * * *$ \\
\hline Variance & $0.23 * * *$ & $0.06 * * *$ & $0.001 * * *$ \\
\hline \multicolumn{4}{|c|}{ 2-Class Solution } \\
\hline \multicolumn{4}{|c|}{ Class $1(n=453)$} \\
\hline Mean & $1.53^{* * *}$ & $0.12 * * *$ & $-0.01 * * *$ \\
\hline Variance & 0.03 & $0.06^{* * *}$ & $0.001^{*}$ \\
\hline \multicolumn{4}{|c|}{ Class $2(n=47)$} \\
\hline Mean & $3.11 * * *$ & $-0.27 *$ & 0.02 \\
\hline Variance & 0.04 & $0.05 * * *$ & $0.001 * *$ \\
\hline
\end{tabular}

mean difference effect sizes (Cohen's $d$ ) were computed at each age. These are presented in Table 4, and indicate large mean differences between the classes in perceived maternal psychological control at most ages, particularly in early adolescence.

For fathers, the 2- and 3-class solutions also emerged as the most feasible according to the indicators (see Table 2). Here again, the 2-class solution was favored because the three class solution involved a third class that represented only $3 \%(n=16)$ of the sample, being both conceptually indefensible and statistically non-robust, as well as a nonsignificant LMR value. The 2-class solution showed a similar classification pattern as the mother solution. Specifically, Class 1 fathers $(n=453,91 \%)$ showed low initial levels of psychological control at age 12 , which increased over time and then leveled off around mid-to-late adolescence (positive slope, negative quadratic term). These fathers comprised the "low increasing/normative" group. Class 2 fathers $(n=47,9 \%)$ showed a slightly higher intercept that decreased steadily over time (negative slope, non-significant quadratic term). These fathers comprised the "moderate decreasing" group (see Fig. 1b for plots; see Table 3 for parameter estimates). Mean differences in perceived fathers' psychological control and their respective effect sizes are displayed in Table 4, and indicate large differences in early adolescence, which are reduced to moderate differences by late adolescence.
Because the classification counts were nearly identical in the mother and father solutions, follow up analyses were necessary to determine if these elevated classes (class 2) were mostly comprised of the same adolescents (i.e., those reporting both mothers and fathers as relatively high in psychological control), rendering separate mother and father models redundant. In total, 17 adolescents fit this description (i.e., classified in Class 2 for both father and mother solutions), meaning the majority who were classified in these classes were unique to either the mothers' class 2 $(60 \%)$ or the father's class $2(65 \%)$, but not both. Therefore, both models were retained.

\section{Parent- and child antecedents of class membership}

The second phase of the analyses was to identify earlyadolescent antecedents of class membership. Both parent- and child-driven effects were examined, specifically the focal parents' self-reported depressive symptoms and parentreported adolescent externalizing behaviors, both at age 12 . Included in the model were demographic covariates for sex, ethnic minority status, parent education level, and family structure. For mothers' psychological control, adolescents in the "moderate stable" class had mothers who reported higher levels of depressive symptoms at age 12 compared to mothers of adolescents in the "low increasing/normative" group (OR $=1.49, p=0.02$ ). These adolescents also had mothers with lower formal education $(\mathrm{OR}=0.25, p=0.05)$. Neither adolescent externalizing behaviors at age 12 , sex, parent education, nor family structure significantly distinguished class membership. In the father model, adolescents in the "moderate decreasing" group were more likely to be male $(\mathrm{OR}=$ $1.05, p=0.04)$ and be a non-white ethnic minority $(\mathrm{OR}=$ $-1.59, p=0.002)$. Adolescents' sex, fathers' education, family structure, father's depressive symptoms at age 12 , and adolescent externalizing behaviors at age 12 did not predict class membership in father psychological control.

\section{Class differences in development of depressive and anxiety symptoms}

The third and final phase of the analyses involved the comparison of adolescents with distinct trajectories of perceived psychological control on their development of depressive and anxiety symptoms from ages 14 to 19 . In a first step, the best-fitting growth solutions were derived for depressive and anxiety symptoms using a model building approach. These models were estimated separately, and each indicated a quadratic solution to be best-fitting. Final models showed positive, curvilinear growth in both depressive and anxiety symptoms from ages 14-19 (see Table S2 in supplemental material for fit indices and parameter estimates). 
Table 4 Effect size estimates in depressive and anxiety symptoms for the distinct psychological control classes

\begin{tabular}{|c|c|c|c|c|c|c|c|c|}
\hline \multirow[t]{2}{*}{ Age } & \multicolumn{4}{|c|}{ Mothers' psychological control } & \multicolumn{4}{|c|}{ Fathers' psychological control } \\
\hline & $M$ normative class & $\begin{array}{l}M \text { moderate } \\
\text { stable class }\end{array}$ & Pooled $S D$ & Cohen's $d$ & $M$ normative class & $M$ moderate decreasing class & Pooled $S D$ & Cohen's $d$ \\
\hline \multicolumn{9}{|c|}{ Psyc. Cont. } \\
\hline 12 & 1.60 & 3.22 & 0.52 & 3.13 & 1.55 & 3.11 & 0.44 & 3.54 \\
\hline 13 & 1.71 & 3.16 & 0.57 & 2.55 & 1.64 & 2.97 & 0.58 & 2.29 \\
\hline 14 & 1.81 & 3.12 & 0.62 & 2.10 & 1.72 & 2.75 & 0.62 & 1.66 \\
\hline 15 & 1.91 & 3.26 & 0.68 & 1.97 & 1.79 & 2.63 & 0.71 & 1.18 \\
\hline 16 & 1.97 & 2.91 & 0.78 & 1.21 & 1.81 & 2.26 & 0.73 & 0.61 \\
\hline 17 & 2.03 & 2.79 & 0.82 & 0.93 & 1.83 & 2.07 & 0.76 & 0.31 \\
\hline 18 & 2.05 & 2.65 & 0.80 & 0.75 & 1.86 & 2.23 & 0.79 & 0.47 \\
\hline 19 & 1.97 & 2.50 & 0.77 & 0.69 & 1.79 & 2.22 & 0.78 & 0.55 \\
\hline \multicolumn{9}{|c|}{ Dep. Symp. } \\
\hline 14 & 1.64 & 2.04 & 0.51 & 0.78 & 1.65 & 1.87 & 0.53 & 0.42 \\
\hline 15 & 1.69 & 2.16 & 0.56 & 0.84 & 1.70 & 1.97 & 0.57 & 0.47 \\
\hline 16 & 1.79 & 2.13 & 0.63 & 0.54 & 1.80 & 1.92 & 0.64 & 0.19 \\
\hline 17 & 1.77 & 2.18 & 0.57 & 0.71 & 1.79 & 1.94 & 0.58 & 0.25 \\
\hline 18 & 1.76 & 2.06 & 0.58 & 0.52 & 1.77 & 1.96 & 0.60 & $\mathbf{0 . 3 3}$ \\
\hline 19 & 1.75 & 1.97 & 0.55 & 0.40 & 1.76 & 1.88 & 0.56 & 0.20 \\
\hline \multicolumn{9}{|c|}{ Anx Symp. } \\
\hline 14 & 0.89 & 1.35 & 0.56 & 0.84 & 0.91 & 1.04 & 0.57 & 0.23 \\
\hline 15 & 0.95 & 1.40 & 0.60 & 0.74 & 0.98 & 1.12 & 0.62 & 0.24 \\
\hline 16 & 1.08 & 1.36 & 0.63 & 0.46 & 1.10 & 1.15 & 0.63 & 0.09 \\
\hline 17 & 1.15 & 1.45 & 0.65 & 0.47 & 1.17 & 1.19 & 0.65 & 0.03 \\
\hline 18 & 1.16 & 1.50 & 0.69 & 0.49 & 1.17 & 1.35 & 0.69 & 0.26 \\
\hline 19 & 1.20 & 1.50 & 0.71 & 0.43 & 1.21 & 1.37 & 0.71 & 0.23 \\
\hline
\end{tabular}

Psyc Cont = Psychological Control; Dep. Symp. = Depressive Symptoms; Anx Symp. = Anxiety Symptoms; $M=$ Mean, $S D=$ Standard Deviation

Then, a prediction model was tested in which individuals' posterior probabilities for being in the elevated class 2 ("moderate stable" for mothers; "moderate decreasing" for fathers) were entered as predictors of the intercept, slope, and quadratic terms. Because there were only two classes (similar to a dummy variable), the associated regression coefficient represented the predicted difference on each growth parameter (intercept, slope, quadratic term) between adolescents in the normative and elevated classes. Importantly, covariates for adolescents' sex, ethnic minority status, parents' education, and family structure were included. These prediction models were estimated twice, once centered at age 14 and again at age 19 , to check in with the growth process at its beginning and end.

Table S3 (supplemental material) contains the results of these analyses, and Figs. 2 and 3 display the growth curves of depressive and anxiety symptoms differentially for the psychological control classes in the mother and father models. In the mother models examining depressive symptoms, membership in the elevated psychological control trajectory ("moderate stable") positively predicted the intercept at age 14 as well as at age 19, but did not predict the slopes or intercepts at these ages. Similar findings were found for the model for anxiety symptoms: membership in the "moderate stable" trajectory positively predicted the intercepts at age 14 and 19, although it was unassociated with slopes and quadratic terms. Interpreted, adolescents in the moderate-stable class on maternal psychological control showed similar rates and patterns of change in depressive and anxiety symptoms from age 14 to 19 as those in the low-increasing/normative class (non-significant prediction of slope and quadratic terms). However, adolescents in the moderate-stable group began their trajectories of depressive and anxiety symptoms significantly higher at age 14 and this difference was sustained through age 19, meaning they also ended higher at age 19 (significant prediction of age 14 and age 19 intercepts). Effect size calculations showed that mean differences in depressive symptoms between the two psychological control groups were large at age $14(d=$ $0.80)$ and moderate by age $19(d=0.40)$. Class differences in anxiety symptoms were also large at age $14(d=0.84)$ and moderate by age $19(d=0.43)$.

Results in the father model were different. In both models (depressive symptoms and anxiety symptoms), classification was associated only with the age 14 intercept. The age 19 intercepts, as well as the slopes and quadratic terms at both ages, were not predicted by class membership. Thus, adolescents in the elevated psychological control trajectory ("moderate-declining") showed higher initial levels of depressive and anxiety symptoms at age 14 than those in the "low-increasing/normative" group, but these differences were not sustained through age 19. That is, 

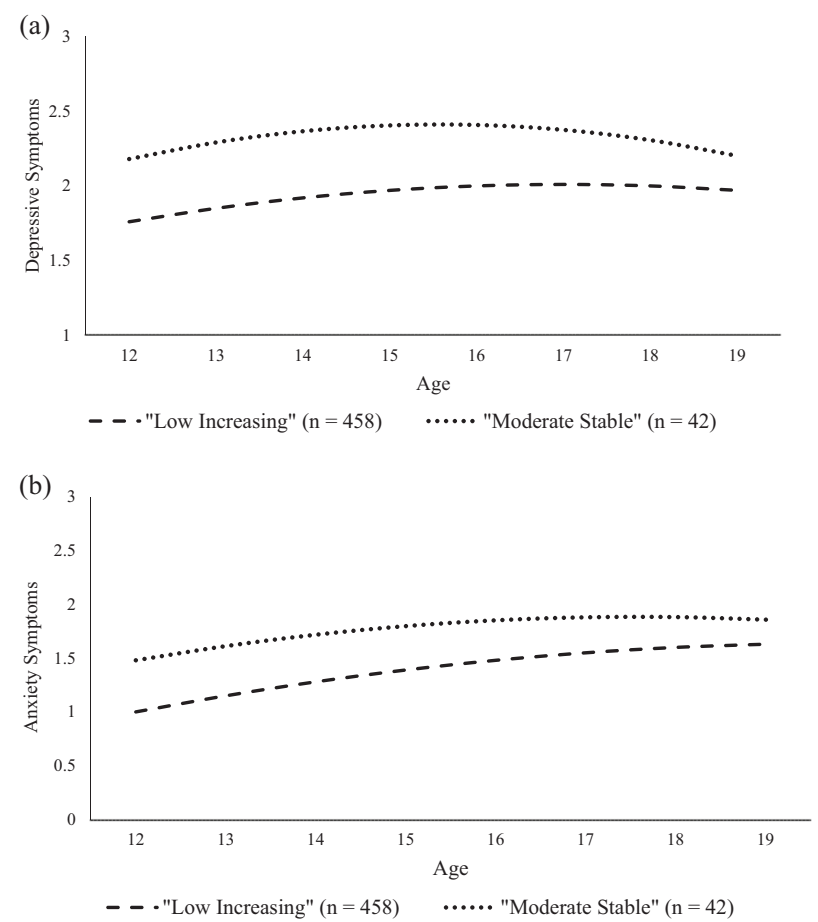

Fig. 2 Trajectories of (a) depressive symptoms and (b) anxiety symptoms from ages 14-19 for the mother psychological control classes
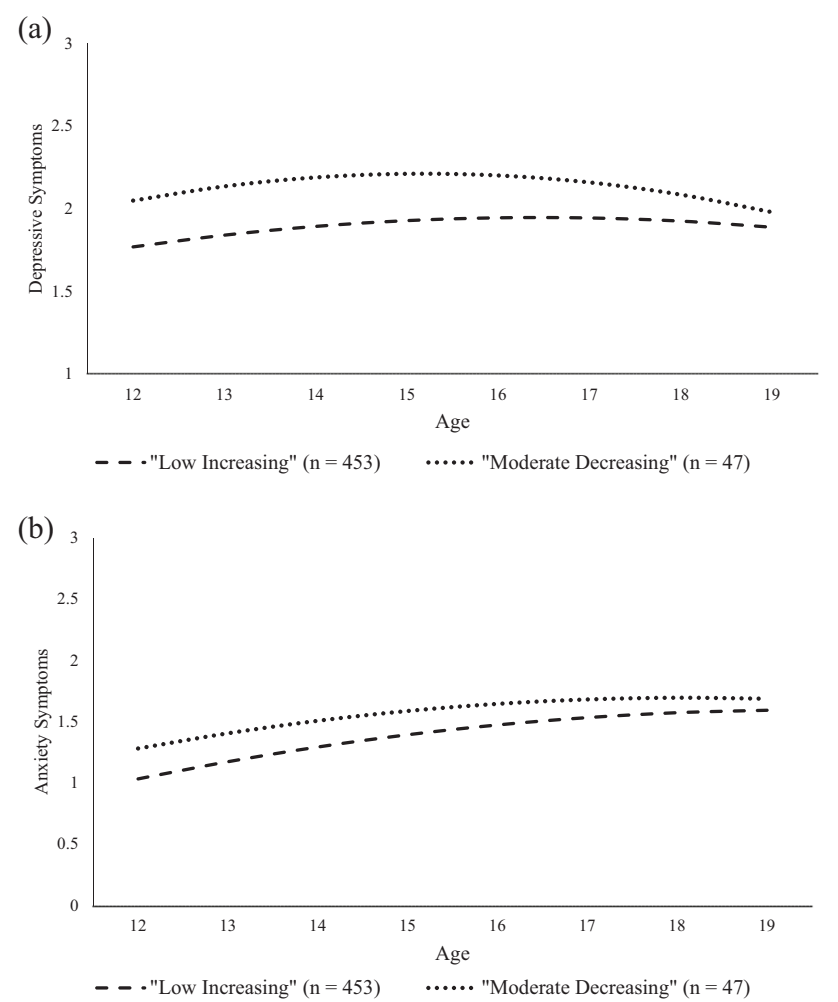

Fig. 3 Trajectories of (a) depressive symptoms and (b) anxiety symptoms from ages 14 to 19 for the father psychological control classes while the "moderate-decreasing" adolescents began higher in their depressive and anxiety symptoms at age 14 than those in the "low-increasing/normative" group, the trajectories of these two classes ultimately converged over time, such that by age 19 , there were no significant differences in anxiety and depressive symptoms between the two classes. Effect size calculations showed that class differences in depressive symptoms were moderate at age $14(d=0.40)$, but relatively small by age $19(d=0.23)$. For anxiety symptoms, class differences were small at age $14(d=$ $0.23)$, and negligible by age $19(d=0.09)$.

\section{Discussion}

Adolescence is a developmental period when the parentchild relationship undergoes normative transition, in part due to the child's increasing need for autonomy and independence (Soenens et al. 2019). This often results in decreases in parental knowledge and effectiveness in attempts to directly control children's behavior (e.g., Keijsers and Poulin 2013), which may open the door for increases in more subtle forms of control such as psychological control. Taking into consideration the consistent negative correlates of psychological control, especially for adolescents and those transitioning to adulthood (e.g., Werner et al. 2016), it is important to explore the development of this behavior across adolescence. In addition, it is informative to consider variability in psychological control and antecedents and consequences of this variability. Thus, the current study sought to examine trajectories of adolescents' perceptions of parental psychological control across adolescence and its significance for adolescents' development of depressive and anxiety symptoms.

\section{Developmental trends in parental psychological control across adolescence}

The first aim of this study was to examine developmental patterns in adolescents' experiences of parental psychological control from early to late adolescence, and to describe heterogeneity (i.e., distinct classes) in this development. Consistent with hypotheses, adolescent-reported psychological control started at relatively low levels during early adolescence and gradually increased until about age 17, after which it leveled off. These increasing experiences of psychological control may be explained in part by children's increasing autonomy that motivates decreases in parents' behavioral control across adolescence (Keijsers and Poulin 2013), despite continued parenting stress (Steeger and Gondoli 2013). Alternatively, as children move toward independence and push away from parents, it is possible that parenting behaviors that may not have felt controlling 
during childhood begin to be perceived differently. Both these processes may come to an end during late adolescence as children prepare to leave the parental home and as the family system more fully calibrates the transition to autonomy. Indeed, the leveling off of psychological control at age 17 found in the current study is consistent with research on psychological control during the transition to adulthood, which has found stable (Luyckx et al. 2007) or decreasing (Desjardins and Leadbeater 2017) levels of perceived psychological control from ages 18 to 25 .

While this overall trend in perceived psychological control is meaningful, the findings also suggested meaningful variability in this trajectory over time, suggesting that not all adolescents perceive their parents similarly during this transition. Notably, for the vast majority of both mothers and fathers (roughly 90\%), trajectories followed the normative pattern described above (low initial levels, slightly increasing over time and then leveling out at age 17). However, nearly $10 \%$ of mothers were reported to exhibit moderate and stable levels of psychological control over time, and nearly $10 \%$ of fathers were reported as showing moderate and decreasing levels over time. Thus, findings support at least a degree of heterogeneity that is not captured in traditional variable-centered approaches. Though $10 \%$ is a clear minority, it nevertheless identifies a meaningful subset of teens who may be at increased risk for negative developmental outcomes. This number is slightly more salient when considering that there was relatively little overlap between the adolescents who reported elevated levels of psychological control from both mother and father: closer to $15 \%$ of adolescents perceived non-normative levels of psychological control from at least one parent. This finding led to the second and third questions, which explored both antecedents and mental health correlates of these different trajectories.

\section{Antecedents of following distinct psychological control trajectories}

The second aim of this study was to identify antecedents of psychological control trajectories. Hypotheses that parent depressive symptoms and child externalizing behaviors would differentially predict class membership were partially supported. Mothers' (but not fathers') depressive symptoms at age 12 were associated with a higher likelihood of being in the moderate-stable class. It is well established that mothers struggling with symptoms of depression display lower quality parenting (Makol et al. 2019), and these mothers may resort to reactive psychologically controlling parenting to maintain control in family life (Pettit et al. 2001). Mothers who experience depressive symptoms also have lower levels of empathy and perspective taking (Werner et al. 2016), both of which are key cognitive and emotional skills necessary for understanding a child's need for autonomy and connection. Thus, maternal depressive symptoms might explain the higher and consistent levels of perceived psychological control in the moderate stable group that was not present in the moderate decreasing group for fathers. If parents have the emotional resources to perceive their adolescents' desires for independence, they may be able to adapt and reduce levels of psychological control in more developmentally appropriate ways over time. However, contrary to hypotheses, there was not a significant link between adolescents' age 12 externalizing behaviors and their psychological control trajectory classification. This may be because the current sample was relatively welladjusted and so levels of externalizing behaviors might not have been high enough to detect this pattern. Future research should consider populations that are at greater risk so predictors of being in this high-risk group are more salient.

\section{Developmental significance of class membership for depressive and anxiety symptoms}

The third aim of this study was to explore the developmental significance of occupying distinct psychological control trajectories, looking particularly at adolescents' development of depressive and anxiety symptoms. Hypotheses were supported for mothers: although mothers in both classes had adolescents with similar rates of increase in depressive and anxiety symptoms from ages 14 to 19 , the mothers in the moderate stable group had adolescents who were significantly elevated at all ages from early to late adolescence. Furthermore, the effect sizes for these differences in the mother models were quite robust and indicated that these differences were large to moderate in size. However, fathers who were in the moderate declining group had adolescents with higher levels of depressive and anxiety symptoms at age 14 (small to moderate effect sizes), but this difference decreased over time and was no longer significant when the teens were age 19 (small to negligible effect sizes). Taken together, this suggests that a small group of mothers (but not fathers) are perceived to display a pattern of parenting that consists of moderate and consistent levels of psychological control over time, which is associated with higher levels of internalizing problems across all adolescence. The father model, however, suggests that if experiences of psychological control decrease over time, so do internalizing problems. This difference may have less to do with the sex of the parent and more to do with the different patterns seen over time between mothers and fathers. Clearly the adolescents who are at the greatest risk are those who perceive relatively high and sustained experiences of psychological control that co-occur with anxiety and depressive symptoms. 
Intervention efforts should pay particular attention to subgroups who may share these risks.

Psychologically controlling parents create a family environment that undermines adolescents' self-determined development of autonomy (e.g., Deci and Ryan 2012), failing to enhance the psychological resources needed to thrive during adolescence (e.g., Soenens and VanSteenkiste 2010). Research has consistently shown that adolescents' experiences of psychological control are associated with higher levels of internalizing problems during adolescence (Soenens et al. 2008), though most studies are cross-sectional or shortterm longitudinal. The current study adds to these findings by showing enduring associations between experiencing psychologically controlling parenting and the development of internalizing symptoms from early to late adolescence. While acknowledging that direction of effects cannot be determined, theory (Deci and Ryan 2012) and empirical work (Rogers et al., under review) certainly support the role that coercive parenting can play in undermining adolescents' healthy emotional development. It is also likely that children who struggle elicit higher levels of parenting stress and less than optimal parenting (e.g., Soenens et al. 2019). Very few studies have explored the role of adolescent mental health on future parenting, and research is needed to more clearly understand this dynamic developmental process.

\section{Limitations}

Though the current study benefitted from the strength of a long-term longitudinal design, there were nevertheless limitations. First, given one of the central goals of the study was to detect heterogeneity in parenting, the current study was limited by a relatively homogeneous sample in regards to income and ethnicity. Future research should seek to replicate the current findings with more disadvantaged and marginalized populations where family stressors may be more prominent and variability in perceptions of parenting may be greater. Second, reports of psychological control, depressive symptoms, and anxiety symptoms were all based on adolescents' self-reports, which suggests findings may be prone to shared method variance. Though adolescents are arguably the most important reporters of negative parenting (Van Lissa et al. 2019) and their own internal states such as depression and anxiety, future research should consider additional reporters (e.g., Makol et al. 2019). The inclusion of parentreports, for example, could lead to richer insights in understanding how psychological control processes change over time. Finally, as noted above, direction of effects could not be determined between psychological control and antecedents or consequences. Though constructs were measured at different time points in the current study, future research should utilize additional longitudinal models that allow for the measurement of bidirectional change over time.

\section{Conclusion}

Parents' abilities to directly control and regulate adolescent behavior generally decline across adolescence. However, very little is understood about parents' more subtle control strategies, including psychological control, which may become more relevant and feasible as adolescents grow in independence. The current study examined trajectories of adolescents' perceived parental psychological control from ages 12 to 19 and explored the developmental significance of these trajectories by examining links to depressive and anxiety symptoms. The study found that the majority of adolescents reported low but increasing levels of parents' psychological control across the second decade. A minority of adolescents, however, experienced relatively elevated and stable levels of psychological control during this time, and reported higher levels of depressive and anxiety symptoms across development. This study offers greater insight to the changing dynamics of parental control across adolescence. While more direct forms of parental control (e.g., monitoring, knowledge) are declining across the adolescent years, adolescents may actually experience steady increases in parents' psychological control, and this trend appears to hold developmental significance for the adolescent.

Authors' Contributions AR conceived of the study, performed the statistical analyses and interpretation of results, and led the writing of the manuscript; LPW conceived of the study and assisted in the interpretation of results and the writing of the manuscript; RM assisted with data analysis and writing of the manuscript; JH assisted with data analysis and writing of the manuscript. All authors read and approved the final manuscript.

Data Sharing and Declaration This manuscript's data will not be deposited.

\section{Compliance with Ethical Standards}

Conflict of Interest The authors declare that they have no conflict of interest.

Ethical Approval All procedures involving human participants were performed in accordance with the ethical standards of the institution and/or national research committee and with the 1964 Helsinki declaration and its later amendments or comparable ethnical standards.

Informed Consent All adolescents and parents in the study assented to participation; consent was obtained from each participants' primary caregiver.

Publisher's note: Springer Nature remains neutral with regard to jurisdictional claims in published maps and institutional affiliations.

\section{Appendix A}

Table 5 
Table 5 Correlations among key variables at age 12 (and age 14 for adolescent depressive and anxiety symptoms)

\begin{tabular}{|c|c|c|c|c|c|c|c|c|}
\hline & 1 & 2 & 3 & 4 & 5 & 6 & 7 & 8 \\
\hline 1. Mother psych. control & - & & & & & & & \\
\hline 2. Father psych. control & $0.62 * * *$ & - & & & & & & \\
\hline 3. Mother depressive symptoms & $0.12 *$ & 0.07 & - & & & & & \\
\hline 4. Father depressive symptoms & $0.13 *$ & $0.15^{*}$ & $0.21 * * *$ & - & & & & \\
\hline 5. Adolescent externalizing behaviors & $0.18 * * *$ & $0.10^{*}$ & $0.11 *$ & 0.07 & - & & & \\
\hline 6. Adolescent depressive symptoms & $0.23 * * *$ & $0.16^{* *}$ & $0.19 * * *$ & $0.15^{*}$ & $0.24 * * *$ & - & & \\
\hline 7. Adolescent anxiety symptoms & $0.20 * * *$ & $0.13 *$ & 0.02 & -0.02 & 0.03 & $0.50 * * *$ & - & \\
\hline 8. Family income & -0.01 & -0.04 & $-0.24 * * *$ & $-0.10 *$ & $-0.10^{*}$ & -0.07 & 0.01 & - \\
\hline
\end{tabular}

$* p<0.05, * * p<0.01, * * p<0.001$

\section{References}

Akaike, H. (1973). Information theory and an extension of the maximum likelihood principle. In B. N. Petrov \& F. Csaki (Eds), Second international symposium on information theory (pp. 267-281). Budapest: Academiai Kiado.

Akaike, H. (1981). Likelihood of a model and information criteria. Journal of Econometrics, 16(1), 3-14. https://doi.org/10.1016/ 0304-4076(81)90071-3.

Asparouhov, T., \& Muthén, B. (2012). Using Mplus TECH11 and TECH14 to test the number of latent classes. Mplus Web Notes, $14,22$.

Barber, B. K. (1996). Parental psychological control: revisiting a neglected construct. Child Development, 67(6), 3296-3319. https://doi.org/10.2307/1131780.

Barber, B. K., Stolz, H. E., Olsen, J. A., \& Maughn, S. L. (2005a). Parental support, psychological control, and behavioral control: assessing relevance across time, culture, and method. Monographs of the Society for Research in Child Development, 70(4). https://doi.org/10.1111/j.1540-5834.2005.00369.x.

Barber, B. K., Stolz, H. E., Olsen, J. A., \& Maughan, S. L. (2005b). Responsiveness, psychological control, and behavioral control: assessing relevance across time, method, and culture. Monographs of the Society for Research in Child Development, 70 (7, Serial No. 276).

Cummings, M. E., Keller, P. S., \& Davies, P. T. (2005). Toward a family process model of maternal and paternal depressive symptoms: exploring multiple relations with child and family functioning. Journal of Child Psychology and Psychiatry, 46(5), 479-789. https://doi.org/10.1111/j.1469-7610.2004.00368.x.

De Los Reyes, A., Goodman, K. L., Kliewer, W., \& Reid-Quiñones, K. (2009). The longitudional consistency of mother-child reporting discrepancies and parental monitoring and their ability to predict child delinquent behaviors two years later. Journal of Youth and Adolescence, 39(12), 1417-1430. https://doi.org/10. 1007/s10964-009-9496-7.

Deci, E. L., \& Ryan, R. M. (2012). Self-determination theory. In P. A. M. Van Lange, A. W. Kruglanski \& E. T. Higgins (Eds), Handbook of theories of social psychology (pp. 416-433). Thousand Oaks, CA: Sage.

Desjardins, T., \& Leadbeater, B. J. (2017). Changes in parental emotional support and psychological control in early adulthood: direct and indirect associations with educational and occupational adjustment. Emerging Adulthood, 5(3), 177-190. https://doi.org/ $10.1177 / 2167696816666974$

Dietvorst, E., Hiemstra, M., Hillegers, M. H., \& Keijsers, L. (2018). Adolescent perceptions of parental privacy invasion and adolescent secrecy: an illustration of Simpson's paradox. Child Development, 89(6), 2081-2090. https://doi.org/10.1111/cdev.13002.
Dishion, T. J., \& McMahon, R. J. (1998). Parental monitoring and the prevention of child and adolescent problem behavior: a conceptual and empirical formulation. Clinical Child and Family Psychology Review, 1(1), 61-75. https://doi.org/10.1023/A: 1021800432380.

Enders, C. K. (2010). Applied missing data analysis. New York, NY: Guilford Press.

Essau, C. A., Muris, P., \& Ederer, E. M. (2002). Reliability and validity of the Spence Children's Anxiety Scale and the Screen for Child Anxiety Related emotional disorders in German children. Journal of Behavior Therapy and Experimental Psychiatry, 33(1), 1-18. https://doi.org/10.1016/S0005-7916(02)00005-8.

Grimm, K. J., Ram, N., \& Estabrook, R. (2017). Growth modeling: Structural equation and multilevel modeling approaches. New York, NY: Guilford Publications.

Janssens, A., Van Den Noortgate, W., Goossens, L., Colpin, H., Verschueren, K., Claes, S., \& Van Leeuwen, K. (2017). Externalizing problem behavior in adolescence: parenting interacting With DAT1 and DRD4 genes. Journal of Research on Adolescence, 27(2), 278-297. https://doi.org/10.1111/jora.12271.

Keijsers, L., \& Poulin, F. (2013). Developmental changes in parent-child communication throughout adolescence. Developmental Psychology, 49(12), 2301-2308. https://doi.org/10.1037/a0032217.

Keijsers, L., Voelkle, M. C., Maciejewski, D., Branje, S., Koot, H., Hiemstra, M., \& Meeus, W. (2016). What drives developmental change in adolescent disclosure and maternal knowledge? Heterogeneity in within-family processes. Developmental Psychology, 52(12), 2057-2070. https://doi.org/10.1037/dev0000220.

King, K. M., Littlefield, A., McCabe, C., Mills, K. L., Flournoy, J., \& Chassin, L. (2017). Longitudinal modeling in developmental neuroimaging research: common challenges, and solutions from developmental psychology. Developmental Cognitive Neuroscience, 33, 54-72. https://doi.org/10.1016/j.den.2017.11.009.

Little, R. J. (1988). A test of missing completely at random for multivariate data with missing values. Journal of the American Statistical Association, 83(404), 1198-1202. https://doi.org/10.1080/ 01621459.1988 .10478722$.

Luyckx, K., Soenens, B., Vansteenkiste, M., Goossens, L., \& Berzonsky, M. D. (2007). Parental psychological control and dimensions of identity formation in emerging adulthood. Journal of Family Psychology, 21(3), 546-550. https://doi.org/10.1037/ 0893-3200.21.3.546.

Makol, B. A., De Los Reyes, A., Ostrander, R. S., \& Reynolds, E. K. (2019). Parent-Youth divergence (and convergence) in reports of youth internalizing problems in psychiatric inpatient care. Journal of Abnormal Child Psychology. https://doi.org/10.1007/ s10802-019-00540-7

Meter, D. J., Ehrenreich, S. E., \& Underwood, M. K. (2019). Relations between parent psychological control and parent and adolescent 
social aggression. Journal of Child and Family Studies, 28(1), 140-151. https://doi.org/10.1007/s10826-018-1240-z.

Muthén, L. K., \& Muthén, B. O. (1998-2018). Mplus user's guide. Seventh edition Los Angeles: Muthén \& Muthén.

Pettit, G. S., Laird, R. D., Dodge, K. A., Bates, J. E., \& Criss, M. M. (2001). Antecedents and behavior-problem outcomes of parental monitoring and psychological control in early adolescence. Child Development, 72(2), 583-598. https://doi.org/10.1111/14678624.00298.

Radloff, L. S. (1977). Center for Epidemiological Studies Depression Scale. PsycTESTS. https://doi.org/10.1037/t02942-000

Rogers, A. A., Memmott-Elison, M. K., Padilla-Walker, L. M., \& Byon, J. (under review). Perceived parental psychological control predicts intra-individual decrements in self-regulation across adolescence.

Smetana, J. G., Metzger, A., Gettman, D. C., \& Campione-Barr, N. (2006). Disclosure and secrecy in adolescent-parent relationships. Child Development, 77(1), 201-217. https://doi.org/10. 1111/j.1467-8624.2006.00865.x.

Soenens, B., Luyckx, K., Vansteenkiste, M., Duriez, B., \& Goossens, L. (2008). Clarifying the link between parental psychological control and adolescents' depressive symptoms: reciprocal versus unidirectional models. Merrill-Palmer Quarterly, 54(4), 411-444. https://doi.org/10.1353/mpq.0.0005.

Soenens, B., \& Vansteenkiste, M. (2010). A theoretical upgrade of the concept of parental psychological control: proposing new insights on the basis of self-determination theory. Developmental Review, 30(1), 74-99. https://doi.org/10.1016/j.dr.2009.11.001.

Soenens, B., Vansteenkiste, M., \& Beyers, W. (2019). Parenting adolescents. In M. Bornstein (Ed.), Handbook of parenting (3rd edtion), Vol. 1: Children and parenting (pp. 101-167). New York, NY: Routledge.

Spence, S. H. (1998). A measure of anxiety symptoms among children. Behaviour Research and Therapy, 36(5), 545-566.

Steeger, C. M., \& Gondoli, D. M. (2013). Mother-adolescent conflict as a mediator between adolescent problem behaviors and maternal psychological control. Developmental Psychology, 49(4), 804-814. https://doi.org/10.1037/a0028599.

Stockings, E., Degenhardt, L., Lee, Y. Y., Mihalopoulos, C., Liu, A., Hobbs, M., \& Patton, G. (2015). Symptom screening scales for detecting major depressive disorder in children and adolescents: a systematic review and meta-analysis of reliability, validity and diagnostic utility. Journal of Affective Disorders, 174, 447-463. https://doi.org/10.1016/j.jad.2014.11.061.

Taraban, L., Shaw, D. S., Leve, L. D., Wilson, M. N., Dishion, T. J., Natsuaki, M. N., Neiderhiser, J. M., \& Reiss, D. (2017). Maternal depression and parenting in early childhood: contextual influence of marital quality and social support in two samples. Developmental Psychology, 53(3), 436-449. https://doi.org/10.1037/ dev0000261.

Van Lissa, C. J., Keizer, R., Van Lier, P. A. C., Meeus, W. H. J., \& Branje, S. (2019). The role of fathers' versus mothers' parenting in emotion-regulation development from mid-late adolescence: disentangling between-family differences from within-family effects. Developmental Psychology, 55(2), 377-389. https://doi. org/10.1037/dev0000612.
Wang, Q., Pomerantz, E.M., Chen, H. (2007) The Role of Parents? Control in Early Adolescents? Psychological Functioning: A Longitudinal Investigation in the United States and China. Child Development 78(5):1592-1610. https://doi.org/10.1111/j.14678624.2007.01085.x.

Weissman, M. M., Orvaschel, H., \& Padian, N. (1980). Children's symptom and social functioning self-report scales: comparison of mothers' and children's reports. Journal of Nervous Mental Disorders, 168(12), 736-740. https://doi.org/10.1097/00005053198012000-00005.

Werner, L. L. A. A., der Graaff, J. V., Meeus, W. H. J., \& Branje, S. J. T. (2016). Depressive symptoms in adolescence: longitudinal links with maternal empathy and psychological control. Journal of Abnormal Child Psychology, 44(6), 1121-1132. https://doi. org/10.1007/s10802-015-0106-8.

Yap, M. B., Allen, N. B., \& Ladouceur, C. D. (2008). Maternal socialization of positive affect: the impact of invalidation on adolescent emotion regulation and depressive symptomatology. Child Development, 79(5), 1415-1431. https://doi.org/10.1111/j. 1467-8624.2008.01196.x.

Adam A. Rogers is an Assistant Professor in the School of Family Life at Brigham Young University. He received his doctorate in 2017 from Arizona State University. His research focuses on family dynamics underlying the development of competence and psychopathology in adolescence, with a particular lens toward gender socialization and development.

Laura M. Padilla-Walker is a Professor in the School of Family Life at Brigham Young University. Her research focuses on the parentchild relationship and media as they relate to prosocial behavior and healthy sexuality.

Ryan D. McLean is a master's student in the School of Family Life at Brigham Young University. His research focuses on parenting during adolescence with an emphasis in understanding tools and techniques to strengthen the parent-child relationship and promote autonomy during the transition to adulthood.

Jeffrey L. Hurst is a student in the psychology department at Brigham Young University. He plans on attending graduate school and becoming a professor. His research interests include parenting that fosters trust through communication. 
Journal of Youth \& Adolescence is a copyright of Springer, 2020. All Rights Reserved. 